$\Rightarrow$ CHROMOSOMES

\section{Keeping centromeric identity}

During cell division, the mitotic spindle attaches to chromosomes at centromeric regions to ensure accurate chromosome segregation. In higher eukaryotes, the centromere is defined by the composition and structure of the chromatin, which in this case contains the histone $\mathrm{H} 3$ variant centromeric protein A (CENPA). The mechanisms underlying CENPA deposition to specify centromere identity are still poorly understood. Now, Kim et al. show that the mammalian MIS18 complex functions by interacting with DNA demethylases DNMT3A and DNMT3B to ensure their centromeric localization and thus the epigenetic states of centromeric chromatin that are required for CENPA loading.

The MIS18 complex has been previously shown to accumulate at the centromere during anaphase to early G1 phase, slightly ahead of CENPA loading, and to be required for the localization of CENPA at centromeres. To assess the physiological role of MIS18, Kim et al.

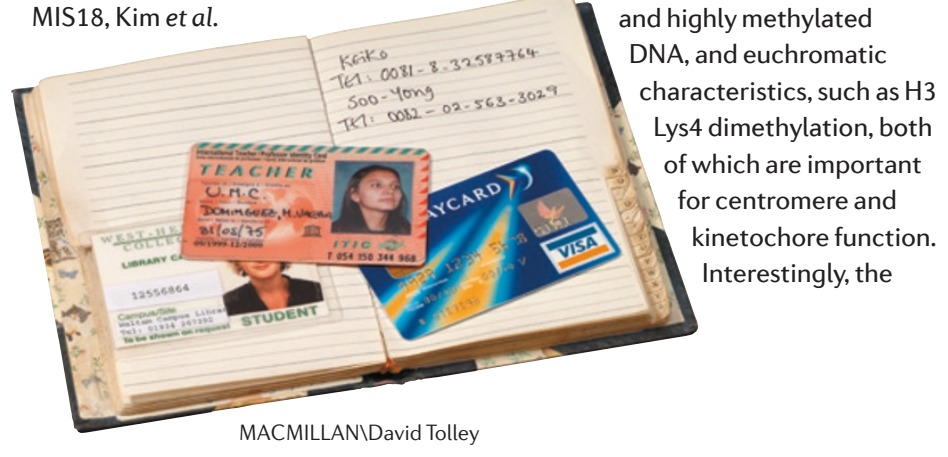
mice for Mis18a (which encodes one of three MIS18 subunits). Knockout embryos died around embryonic day 3.5 and knockout blastocysts grown in vitro showed severe chromosomal missegregation and lack of centromeric CENPA, which ultimately caused cell death. Interestingly, this phenotype is almost identical to that of embryos lacking CENPA, which confirms a functional link between MIS18 $\alpha$ and CENPA.

The authors further investigated the function of MIS18 $\alpha$ in conditional Mis18a knockout mouse embryonic fibroblasts (MEFs). Consistent with their observations in cultured blastocysts, mutant MEFs showed abnormal chromosome segregation and strongly reduced centromeric localization of CENPA. Furthermore, MEFs arrested in mitosis showed defects in the organization of centromeric regions.

Centromeres have both heterochromatic characteristics, such as $\mathrm{H} 3$ Lys 9 trimethylation and highly methylated DNA, and euchromatic haracteristics, such as $\mathrm{H} 3$ thylation, both kinetochore function. Interestingly, the generated conditional knockout authors found that these epigenetic states were altered at the centromere in MEFs lacking MIS18a, which suggests that MIS18a is important to maintain centromeric chromatin states.

Moreover, Kim et al. identified DNMT3A and DNMT3B as MIS18 $\alpha$ interacting proteins. Centromeric localization of DNMT3A and DNMT3B was reduced in Mis18a-deficient MEFs, and, vice versa, knockdown of Dnmt3a and Dnmt $3 b$ reduced MIS18a levels at the centromere. This suggests that MIS18a and the DNA demethylases cooperate to localize at the centromere.

Mis18a was found to interact with these DNA demethylases through a Leu-rich region located at its carboxyl terminus. Importantly, knockout MEFs expressing Mis18a mutated at this C-terminal region were hypomethylated at the centromere and showed defects in CENPA centromeric localization.

Together, these results show that DNMT3A- and DNMT3B-mediated DNA methylation at the centromere is required for centromeric localization of CENPA and that MIS18a interacts with these DNA demethylases at the centromere to ensure their centromeric localization. Thus, these studies reinforce the hypothesis that the MIS18 complex functions to propagate centromeric identity.

Kim Baumann

ORIGINAL RESEARCH PAPER Kim, I. S. et al. Roles of MIS18a in epigenetic regulation of centromeric chromatin and CENP-A loading Mol. Cell 17 Apr 2012 (doi:10.1016/j. molcel.2012.03.021) 